TITLE:

\title{
Effect of crystallographic orientation on tensile fractures of (100) and (110) silicon microstructures fabricated from silicon-on-insulator wafers
}

\section{$\operatorname{AUTHOR}(S)$ :}

Uesugi, Akio; Tsuchiya, Toshiyuki; Tabata, Osamu; Sugano, Koji; Hirai, Yoshikazu

\section{CITATION:}

Uesugi, Akio ... [et al]. Effect of crystallographic orientation on tensile fractures of (100) and (110) silicon microstructures fabricated from silicon-on-insulator wafers. Micro \& Nano Letters 2015, 10(12): 678-682

\section{ISSUE DATE:}

2015-12-01

URL:

http://hdl.handle.net/2433/207673

\section{RIGHT:}

This paper is a postprint of a paper submitted to and accepted for publication in 'Micro \& Nano Letters' and is subject to Institution of Engineering and Technology Copyright. The copy of record is available at IET Digital Library:; This is not the published version. Please cite only the published version.; この論文は出版社版でありません。引用の際には出版社 版をご確認ご利用ください。 


\title{
Effect of crystallographic orientation on tensile fractures of (100) and (110) silicon microstructures fabricated from silicon-on-insulator wafers
}

\author{
A. Uesugi, Y. Hirai, K. Sugano, T. Tsuchiya, and O. Tabata
}

Department of Micro Engineering, Kyoto University, Kyoto 615-8540, Japan

E-mail:a_uesugi@nms.me.kyoto-u.ac.jp

This paper investigates the effect of crystallographic orientation on tensile fractures of silicon microstructures. Specimens $5 \mu \mathrm{m}$ wide and $5 \mu \mathrm{m}$ thick were fabricated on (100) and (110) wafers with $\langle 100\rangle,\langle 110\rangle$, and $\langle 111\rangle$ tensile axes. To explore the effects of different surface orientations and morphologies, these specimens were patterned from (100) and (110) silicon-on-insulator (SOI) wafers using the Bosch process under identical fabrication conditions, while other specimens were fabricated from (110) wafers under different conditions. Tensile tests of specimens prepared under the identical fabrication conditions showed that (100) specimens had lower strength than (110) specimens along <100> and <110> axes; the average strength decreased from $3.62 \mathrm{GPa}$ to $3.14 \mathrm{GPa}$ for $\langle 110\rangle$. This decrease in strength is related to differences in damage that ultimately causes fractures. While (110) specimens fractured due to fabrication damage at top corners, fractures of (100) specimens were due to pit-like defects on bottom surfaces. Since these defects were introduced during SOI bonding processes, the fractures of (100) specimens were dominated by intrinsic SOI defects rather than damage introduced during specimen fabrication processes. To realize higher-strength structures on SOI wafers, both the damage caused during fabrication and the intrinsic defects need to be controlled.

1. Introduction: Silicon is one of the standard materials for fabrication of MEMS (Micro Electro Mechanical Systems). Since MEMS functions depend on mechanical deformations, evaluations of mechanical properties of silicon are essential for improving reliability, and have been widely studied. Values of the elastic modulus measured experimentally in micrometer-sized structures [1,2] agree well with theoretical values, namely, $130 \mathrm{GPa}$ along $\langle 100\rangle, 169 \mathrm{GPa}$ along $\langle 110\rangle$, and $188 \mathrm{GPa}$ along <111>. However, further investigation of fracture properties is required, to improve our understanding of fracture mechanisms and allow predictions of fracture strength, since silicon generally shows brittle fracture behavior and its fracture strength is affected by many factors such as structure size [3,4], fabrication methods [5], crystallographic orientations [6], ambient humidity [7], and temperature [8]. The difficulty of predicting the fracture strength of silicon structures is responsible for the imposition of high safety factors in device designs, which constrains the full exploitation of silicon's excellent material properties.

Silicon-on-insulator (SOI) wafers and the Bosch process are widely used to fabricate MEMS devices. The Bosch deep reactive-ion etching process can fabricate high aspect ratio structures but the fabricated sidewalls typically include periodic undulations, such as the scallops shown in Fig. 1. The sidewall surfaces are damaged as isotropic plasma etching and passivation steps are alternately repeated when creating a structure, and critical locations where surfaces meet may be unevenly etched, such as at the boundaries between the silicon substrate and mask patterns or buried oxide layers. Since these defacements act as fracture origins, the effect of surface damage
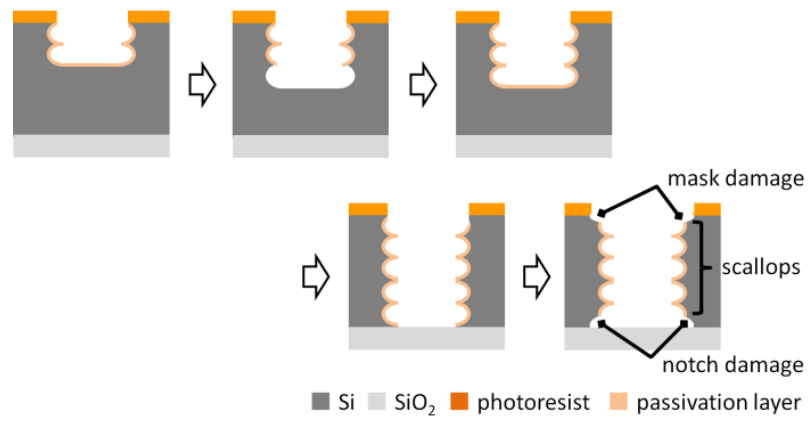

Fig. 1 Surface damage caused by the Bosch process. upon fracture properties $[9,10]$ and post-processing treatment techniques [11-13] have been investigated, in order to improve the reliability of silicon structures.

We also have reported the tensile fracture properties of a micrometer-sized structure prepared with the Bosch process [14]. In order to discuss the effect of crystallographic orientations on tensile fracture properties, we measured specimens $5 \mu \mathrm{m}$ in thickness taken from (110) SOI wafers, with various surface morphologies prepared under different fabrication condition changes and with $\langle 100\rangle,\langle 110\rangle$, and $\langle 111\rangle$ tensile axes. Our investigation revealed that tensile strength depends on two effects, namely, the effect of crystallographic orientation and the effect of surface damage, and that there are quantitative relationships between the observed tensile strengths and the shapes of fractured specimens. In particular, the $\langle 111\rangle$ tensile strengths were found to be inversely proportional to the square root of the lengths of the corner defects measured in plan view, which acted as fracture origins.

To consider the effect of different surface orientations, the current research extends the investigation to include evaluation of (100) silicon, which is commonly used in MEMS fabrication. To improve the reliability of fabricated devices, consistent tendencies in fracture behavior may be revealed by evaluating structures that have various surface orientations. In our research, 5 - $\mu \mathrm{m}$ thick specimens were prepared from (100) SOI wafer, applying the same design on $\langle 100\rangle$ and $\langle 110\rangle$ tensile axes and using the same fabrication conditions as in the previous report [14]. The specimens were subjected to quasi-static tensile $[2,3,7,12,14]$ and tensile fatigue tests, and analyzed with fractography based on scanning electron microscope (SEM) observations. In our comparison of the fracture properties of (100) and (110) silicon, we discuss the effect of crystallographic orientation on tensile fractures of silicon, particularly the fracture criteria for silicon microstructures fabricated from SOI wafer.

2. Experimental Method: Schematic diagrams of layouts used for testing chips are shown in Fig. 2, with plan and elevation views of a specimen appearing in Fig. $2 a$. The free end of each specimen was formed as a large paddle that could be electrostatically gripped when performing tensile strength tests $[2,3,7,12,14]$. The portion of the specimens subjected to testing were $120 \mu \mathrm{m}$ long, $5 \mu \mathrm{m}$ wide and 5 $\mu \mathrm{m}$ thick. Fig. $2 b$ shows two arrangements of the specimens in 4-mm 
(a)

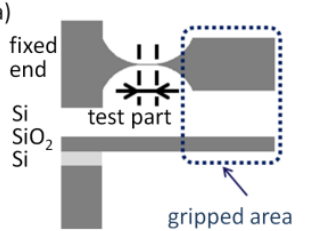

(b)

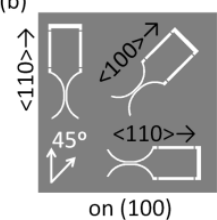

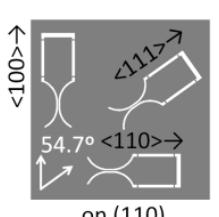

on (110)

Fig. 2 Tensile test specimen designs.

a Plan and elevation views of SOI specimen.

$b$ Specimen arrangements for (100) and (110) testing.

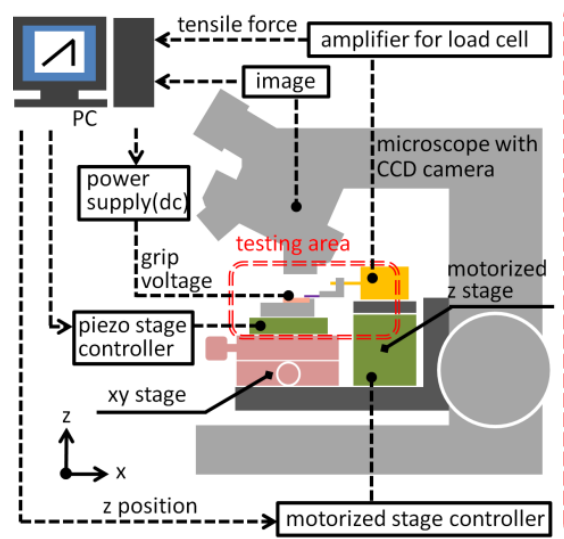

Fig. 3 Block diagram of testing system.

(a)

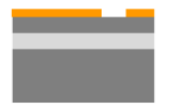

(b)

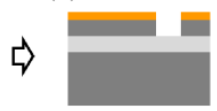

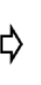

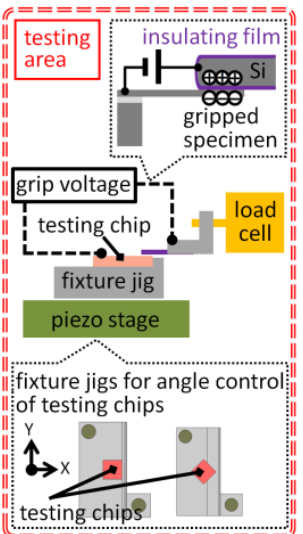

(d)

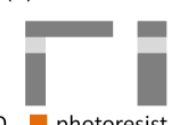

Fig. 4 Specimen fabrication process.

$a$ UV Photolithography.

$b$ Specimen patterning.

$c$ Handle layer removal.

$d$ Sacrificial oxide layer removal.

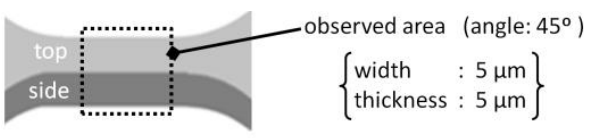

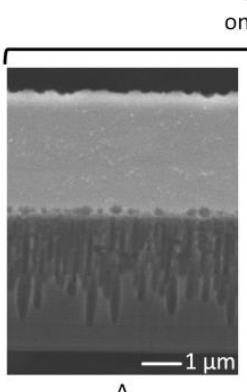

A on $(110)$

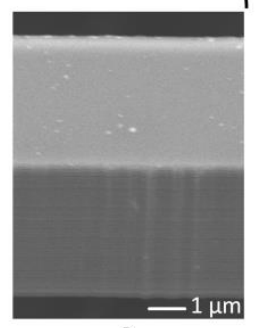

B

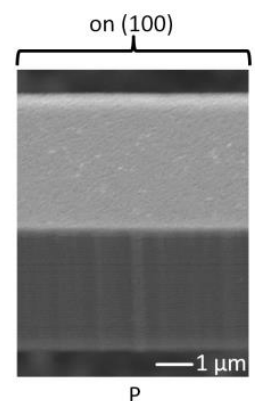

$\mathrm{P}$

Fig. 5 Fabricated specimens' surface morphologies (Angle: $45^{\circ}$ ).

square chips on (100) and (110) wafers, respectively. The specimens were aligned on the major crystallographic orientations, <100>, $\langle 110\rangle$ and $\langle 111\rangle$. By fabricating specimens in different orientations on the same chip, the effects of processing variations were minimized so that any effects due to the different orientations could be clarified.

The tensile testing system is shown in Fig. 3. Tensile force was applied using a piezoelectric nanopositioning stage (piezo stage) and measured with a load cell. The chips being tested were aligned using accurately milled slots provided on the fixture jig (Fig. 3) so that the orientation angle was accurately controlled. During measurements, the

specimen was electrostatically gripped by a silicon probe covered with an insulating film. Tensile strength testing was conducted in laboratory air at pull rate of $1.0 \mu \mathrm{m} / \mathrm{s}$ of the piezo stage. Tensile fatigue testing was performed in a controlled environment, at $25^{\circ} \mathrm{C}$ and $50 \% \mathrm{RH}$. A frequency of $70 \mathrm{~Hz}$ was used for the cyclic loading during tensile fatigue testing.

3. Specimen Fabrication: Three types of specimens were prepared for two silicon tensile fracture evaluations, one focusing on the effect of surface morphology effect and the other on the effect of wafer surface orientation. The fabrication process, shown in Fig 4, consisted of UV photolithography, specimen patterning, handle layer removal, and sacrificial layer removal. The specimen patterning process included a surface treatment process with an isotropic silicon etching solution and the Bosch process. The (110) specimens, called type A and $\mathrm{B}$ when measured in our previous report [14], were prepared so that two different surface morphologies were present, as a result of different surface treatment time of $15 \mathrm{~s}$ and $5 \mathrm{~s}$ for types $\mathrm{A}$ and $\mathrm{B}$, respectively. The (100) specimens used in the current research, called type $\mathrm{P}$, were fabricated under the same fabrication conditions as for type B.

Fig. 5 shows surface morphologies of the fabricated specimens. Comparing the type A and type B specimens, the excess surface treatment given to the type A specimen caused considerable damage at the resist mask edges and severe damage at the intersection of the upper surface and the vertical sidewalls, as well as to the surface of the vertical sidewalls. The specimens fabricated when positioned at different angular orientations showed similar surface morphologies, because crystallographic anisotropies minimally affect the employed fabrication processes.

4. Results: The three types of specimens were subjected to tensile tests. The stage displacement-stress curve illustrated in Fig. 6 is linear and the loading rate was calculated to be $0.14 \mathrm{GPa} / \mathrm{s}$. All three specimens behaved nearly identically, with their stress curves

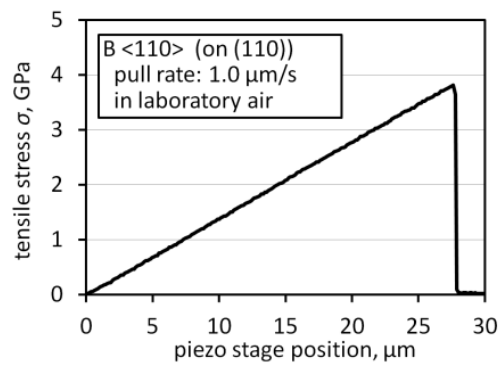

Fig. 6 Relationship between stage displacement and measured stress.

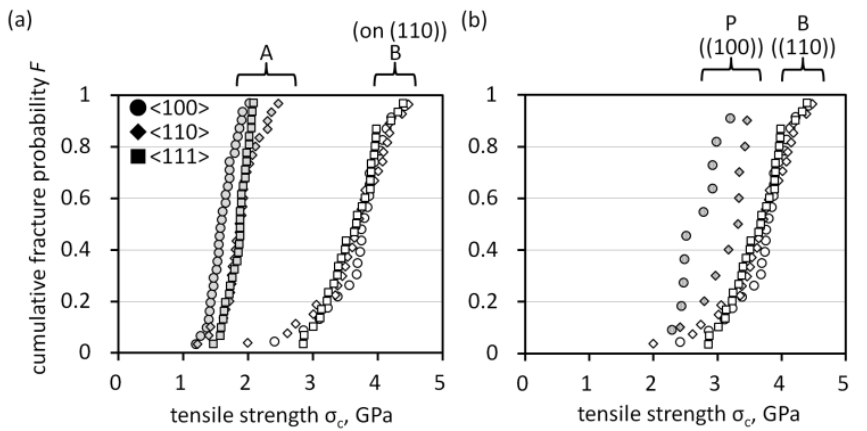

Fig. 7 Cumulative fracture probabilities for the three types of specimen based on the mean rank method.

$a$ (110) specimens with different fabrication conditions.

$b$ (100) and (110) specimens with the same fabrication conditions. 
Table 1: Average strengths, their variations, and Weibull distribution parameters of the three types of specimens.

\begin{tabular}{|c|c|c|c|c|c|c|c|c|c|}
\hline \multirow[b]{2}{*}{ Type } & \multirow{2}{*}{$\begin{array}{c}\text { Surface } \\
\text { orientation }\end{array}$} & \multirow{2}{*}{$\begin{array}{l}\text { Tensile } \\
\text { axis }\end{array}$} & \multirow{2}{*}{$\begin{array}{l}\text { Number of } \\
\text { specimens }\end{array}$} & \multicolumn{2}{|c|}{ Average strength $(\mathrm{GPa})$} & \multicolumn{2}{|c|}{ Average strength variation on each type } & \multicolumn{2}{|c|}{ Weibull distribution parameters } \\
\hline & & & & \begin{tabular}{|l|} 
Orientations \\
\end{tabular} & Total & from total average & from $<110>$ strength & $\begin{array}{c}\text { Scale factor } \\
\sigma_{0}(\mathrm{Gpa})\end{array}$ & $\begin{array}{c}\text { Weibull modulus } \\
m\end{array}$ \\
\hline \multirow{3}{*}{ A } & \multirow{3}{*}{$(110)$} & $\langle 100\rangle$ & 30 & 1.61 & \multirow{3}{*}{1.78} & $-9.9 \%$ & $-14.8 \%$ & 1.70 & 8.94 \\
\hline & & $\langle 110\rangle$ & 29 & 1.89 & & $5.8 \%$ & & 2.01 & 7.10 \\
\hline & & $\langle 111\rangle$ & 30 & 1.86 & & $4.1 \%$ & $-1.6 \%$ & 1.93 & 12.25 \\
\hline \multirow{3}{*}{$\mathrm{B}$} & \multirow{3}{*}{$(110)$} & $\langle 100\rangle$ & 22 & 3.69 & \multirow{3}{*}{3.64} & $1.3 \%$ & $1.9 \%$ & 3.92 & 7.73 \\
\hline & & $\langle 110\rangle$ & 26 & 3.62 & & $-0.6 \%$ & & 3.90 & 6.06 \\
\hline & & $\langle 111\rangle$ & 29 & 3.62 & & $-0.6 \%$ & $0 \%$ & 3.81 & 9.54 \\
\hline \multirow{2}{*}{$\mathrm{P}$} & \multirow{2}{*}{ (100) } & $\langle 100\rangle$ & 10 & 2.70 & \multirow{2}{*}{2.92} & $-7.5 \%$ & $-14.0 \%$ & 2.84 & 8.61 \\
\hline & & $\langle 110\rangle$ & 9 & 3.14 & & $7.5 \%$ & & 3.32 & 7.90 \\
\hline
\end{tabular}

showing sudden drops indicating brittle fractures. Fig. 7 shows distributions of the fracture strengths as cumulative fracture probabilities, and Table 1 summarizes the average strengths and variations. The tensile strengths of the (100) specimens were lower than those of (110) specimens for both $\langle 100\rangle$ and $\langle 110\rangle$; the $\langle 110\rangle$ tensile strength for (100) specimens was smaller by $13 \%$ than that of (110) specimens. The difference in tensile strength was much larger than expected, based on the observed close similarity of surface morphologies and this difference was smaller than that between type $\mathrm{A}$ and $\mathrm{B}$, which has clear different surface morphologies, as shown in Fig. 5. On the other hand, the average tensile strength variation on (100) specimens between $\langle 100\rangle$ and $\langle 110\rangle$ was about $15 \%$, larger than the variation for $\mathrm{B}(110)$ specimens, which was less than $2 \%$ among the three orientations.

The distributions of the tensile strengths were analyzed statistically using Weibull analysis [15], using the following function for cumulative fracture probability:

$$
F=1-\exp \left\{-\left(\frac{\sigma}{\sigma_{0}}\right)^{m}\right\}
$$

where $F, \sigma$, and $\sigma_{0}$ are the cumulative fracture probability, stress and a scale parameter, respectively. $m$ is the Weibull modulus, and higher values of $m$ correspond to smaller distributions. The tensile strength distribution for (100) specimens showed smaller deviation on $\langle 100\rangle$ than on $\langle 110\rangle$. This tendency was similar to the results for (110) specimens, indicating a $m_{<110\rangle}<m_{<100\rangle}<m_{<111>}$ relationship. The fact that $m_{<110\rangle}$ is smaller than $m_{<100\rangle}$ for both (100) and (110) specimens indicates that the strength distribution is related to the crystallographic orientation.

The (100) specimens were also subjected to tensile fatigue testing up to $10^{6}$ cyclic loadings. The maximum stress was set to $90 \%$ of the average strengths, and the stress ratio was 0.05 . As a result, more than $80 \%$ of the specimens survived more than $10^{6}$ cyclic tensile loadings, and no clear differences in fatigue behavior relative to crystallographic orientations were observed. A larger number of loading cycles would be required to investigate such differences.

5. Fractography: Fig. 8 shows fractured type $B$ and type $P$ specimens. The fracture surfaces of both specimens were (111) oriented. Silicon has (110) and (111) planes as cleavage planes but the (111) plane has a smaller surface energy $[16,17]$, which we assume is why the fracture surfaces occurred along the (111) planes. While $\mathrm{B}\langle 110\rangle$ specimens had fracture propagations initially showing on (110) planes, regardless of differences in surface morphology, (110) planes were not clearly observed in any of the $\mathrm{P}<110>$ specimens.

The locations of fracture origins in type $\mathrm{B}$ and type $\mathrm{P}$ specimens were also different. While fractures in type B specimens occurred (a)

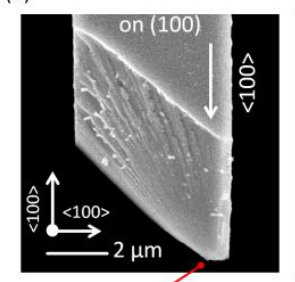

(b)

fracture origin

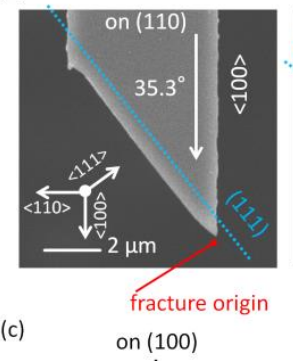

(c)

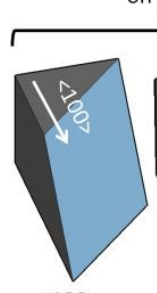

$<100>$

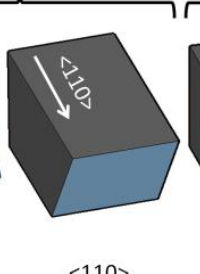

$<110>$

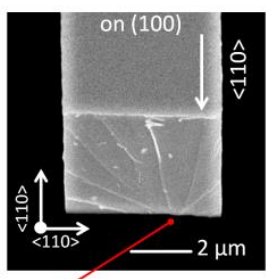

fracture origin

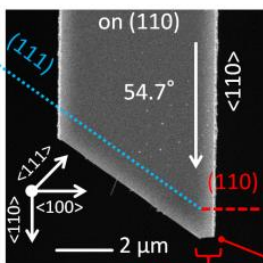

initial area on fracture propagation on (110)

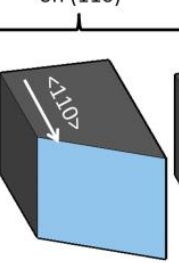

$<110>$

$<111>$
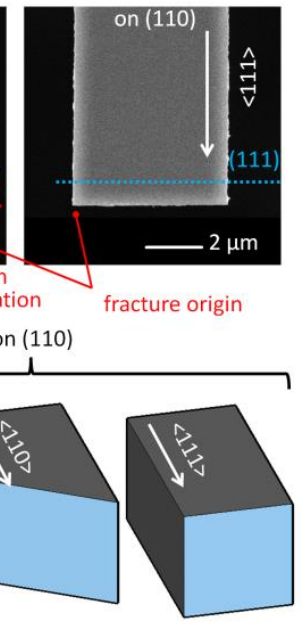

cleavage planes on (111)
Fig. 8 Fracture shapes of the specimens.

$a$ Plan views of (100) specimens (type P).

$b$ Plan views of (110) specimens (type B) [14].

$c$ Schematics of fractured shapes.
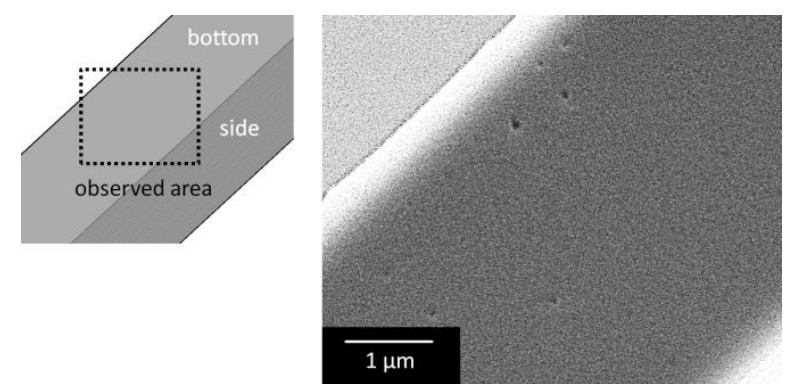

Fig. 9 Surface morphology of bottom surface of (100) silicon. Pit-like defects are scattered on the bottom surface. 
from damage sites located along the top edge, type $\mathrm{P}$ specimen fractures originated from intrinsic bottom surface defects. Fig. 9 shows the pit-like defects present in the bottom surface of a type P specimen, defects that we believe act as fracture origins. Since the defects at interface between the silicon and buried oxide layers of the SOI were intrinsic defects which would be formed by dislocation generated during the SOI bonding process [18], type P fractures were dominated by intrinsic defects rather than the damage caused by etching during the specimen fabrication processes, as shown in Fig.4. This result indicates that as etching damage is reduced, the influence of intrinsic defects increases, and that both of these undesirable influences need to be controlled in order to realize microstructures that exhibit higher fracture strength and great reliability.

A possible reason why the fracturing of type B specimens was due to etching damage rather than intrinsic defects is that the size and shape of the intrinsic defects are different between the type B and type $P$ specimens. Since these intrinsic defects are much affected by the surface condition of the base wafers and the bonding process, the shape and size of the intrinsic defects varies among different wafers, and the effect of these defects particularly depends on the surface orientation. Additionally, the shapes of the intrinsic defects may depend on the orientation of the wafer surface. Generally, intrinsic defects are classified as crystal-originated particles (COP) and the surfaces inside the defects are mainly (111) oriented. The orientation may contribute to the prevalence of sharper pits on (100) surfaces compared with (110) surfaces, which would cause higher stress concentrations to appear on (100) specimens.

6. Discussion: Fig. 10 shows the crystallographic relationship for $\langle 110\rangle$ specimens. Considering the symmetry of silicon's diamond cubic crystal structure, $\langle 110\rangle$ specimens are equivalent for (100) and (110) specimens; $\langle 110\rangle$ specimens are rotated $90^{\circ}$ around the loading axis for the (100) and (110) specimens, so the surface planes consist of (100) and (110) planes, respectively.

Here we review the difference in tensile strength between $B<110>$ and $\mathrm{P}<110>$ specimens. Type $\mathrm{B}$ and type $\mathrm{P}$ specimens fractured from edges and surfaces, respectively, but both of these fracture types originated from (100) surface defects. Therefore, differences in fracture shapes and origins appear to be correlated with differences in tensile strength.

One observed difference in the fracture shapes concerns the initial fracture surface that appears as the fracture propagates; $B<110>$ specimens initially showed (110) fracture planes. In our previous report [14], we showed that higher-strength fractured (110) specimens had smaller (110) plane segments visible in plan view, which agrees with an analytical estimation indicating that quasi-static fracture energy for (110) planes is larger than that for (111) planes under $\langle 110\rangle$ tensile stress. Considering reported surface energy based on density functional theory (DFT); $1.73 \mathrm{~J} / \mathrm{m}^{2}$ for (110) planes and 1.44 $\mathrm{J} / \mathrm{m}^{2}$ for (111) planes [17], and fracture plane angles, dissipated energy for quasi-static fracture under $\langle 110\rangle$ tensile stress can be calculated as $3.46 \mathrm{~J} / \mathrm{m}^{2}$ for $(110)$ planes $\left(=1.73 \mathrm{~J} / \mathrm{m}^{2} \times 2\right)$ and $3.53 \mathrm{~J} / \mathrm{m}^{2}$ for $(110)$ planes $\left(=1.44 \mathrm{~J} / \mathrm{m}^{2} \times 2 / \cos 35.3^{\circ}\right)$. Despite the analytical estimation, the experimental results of type $\mathrm{P}\langle 110\rangle$ specimens showed tensile fractures along (111) planes, rather than along (110) planes. This fact
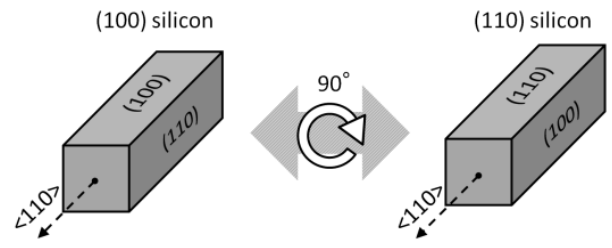

Fig. 10 Crystallographic relationship of $\langle 110\rangle$ specimens. indicates that, due to small difference in the estimated energies required for quasi-static fractures, the initial fracture surface that appears as the fracture propagates is related to a shape of damage that ultimately causes fractures. While the shape of the fabrication damage were rounded due to isotropic plasma etching during the Bosch process, the shapes of the intrinsic defects of type $\mathrm{P}$ specimens were (111) oriented, which would contribute to the initial fracture surface along (111) planes.

The above comparison indicates that the tensile strength of the specimens is profoundly affected by the depth of the defect at which a fracture originates, compared with the difference in initial fracture surfaces. The intrinsic defects and the etching damage on edges were measured using SEM images. The size of the intrinsic defects observed for this report was less than $0.12 \mu \mathrm{m}$ in diameter, while the depth of etching damage reported previously occurred in a range from about 0.06 to $0.15 \mu \mathrm{m}$. If the intrinsic defects are assumed to be COP along (111) surfaces, their depth of roughly $0.08 \mu \mathrm{m}$ can be calculated based on plane angles. The depth of both the intrinsic defects and the etching damage are consistent with the tensile strengths of (100) and (110) specimens; both specimens show a similar range of tensile strengths and certain (110) specimens have higher strength than (100) specimens, as the depths overlapped. This indicates that $\langle 110\rangle$ tensile strengths can be mainly estimated using the depth of defects that lead to fractures, although the locations and shapes of surface damage also affect tensile strength.

7. Conclusion: To investigate the effect of surface orientations on the tensile strength of silicon microstructures, measurements of micrometer-sized structures fabricated from (100) and (110) SOI wafers were carried out along the major crystallographic orientations. Our results showed that the different tensile axes caused average strength variations of less than $15 \%$ for each type of specimen. On the other hand, among the specimens prepared using the same fabrication conditions, the tensile strength of (100) specimens was lower than that of (110) specimens for both $\langle 100\rangle$ and $\langle 110\rangle$. This decrease in tensile strength was affected by differences in the characteristics of the surface defects from which a fracture ultimately originates. (100) specimens fractured from intrinsic SOI defects at the interface between the silicon and buried oxide layers that were unaffected by the specimen fabrication process. This result indicates that intrinsic SOI defects become increasingly important in fracture behavior as size of surface defects occurring during fabrication is decreased. While accurate estimations of strength are currently unavailable, our experimental results indicate that the depth of surface defects is a crucial factor that affects the strength of fabricated silicon. In order to fabricate structures that have higher fracture strengths, the sizes of defects due to fabrication damage as well as intrinsic defects need to be controlled.

8. Acknowledgments: This work was supported by Grant-in-Aid for JSPS Fellows.

\section{Information on References:}

[1] Tsuchiya, T., Hirata, M., Chiba, N., Udo, R., Yoshitomi, Y., Ando, T., Sato, K., Takashima, K., Higo, Y., Saotome, Y., Ogawa, H., and Ozaki, K.: 'Cross comparison of thin-film tensile-testing methods examined using single-crystal silicon, polysilicon, nickel, and titanium films', J. Microelectromech. Syst., 2005, Vol. 14, No. 5, pp. 11781186

[2] Tsuchiya, T., Ikeda, T., Tsunematsu, A., Sugano, K., and Tabata, O.: 'Tensile testing of single-crystal silicon thin films at $600^{\circ} \mathrm{C}$ using infrared radiation heating', Sens. Mater., 2010, Vol. 22, No. 1, pp.111 
[3] Tsuchiya, T., Tabata, O., Sakata, J., and Taga, Y.: 'Specimen size effect on tensile strength of surface micromachined polycrystalline silicon thin films', J. Microelectromech. Syst., 1998, Vol. 7, No. 1, pp. 106-113

[4] Namazu, T., Isono, Y., and Tanaka, T.: 'Evaluation of size effect on mechanical properties of single crystal silicon by nanoscale bending test using AFM', J. Microelectromech. Syst., 2000, Vol. 9, No. 4, pp. 450-459

[5] Yi, T., Li, L., and Kim, C.J.: 'Microscale material testing of single crystalline silicon: process effects on surface morphology and tensile strength', Sens. and Actuators A, 2000, Vol. 83, pp. 172-178

[6] Li, X., Kasai, T., Nakao, S., Ando, T., Shikida, M., Sato, K., Tanaka, H.,: 'Anisotropy in fracture of single crystal silicon film characterized under uniaxial tensile condition', Sens. Actuators A, 2005, Vol. 117, Iss. 1, pp. 143-150

[7] Tsuchiya, T., Yamaji, Y., Sugano, K., and Tabata, O.: 'Tensile and tensile-mode fatigue testing of microscale specimens in constant humidity environment', Experimental Mechanics, 2010, Vol. 50, Iss. 4, pp. $509-516$

[8] Nakao, S., Ando, T., Shikida, M., and Sato, K.: 'Effect of temperature on fracture toughness in a single-crystal-silicon film and transition in its fracture mode' J. Micromech. Microeng., 2008, Vol. 18, Iss. 1, pp. 1-7

[9] Izumi, S. Kubodera, Y. Sakai, S. Miyajima, H. Murakami, and K. Isokawa, T.: 'Influence of ICP Etching Damage on the BrittleFracture Strength of Single-Crystal Silicon' Zairyo/Journal of the Society of Materials Science, Japan, 2007, Vol. 56, Iss. 10, pp. 920925

[10] Michael S. Gaither, Richard S. Gates, Rebecca Kirkpatrick, Robert F. Cook, and Frank W. DelRio: 'Etching process effects on surface structure, fracture strength, and reliability of single-crystal silicon theta-like specimens', J. Microelectromech. Syst., 2013, Vol. 22, No. 3, pp. 589-602

[11] Shikida, M., Niimi, Y., Hasegawa, T., Sugino, T., Hamaoka, S., and Fukuzawa, K.: 'Mechanical strengthening of Si cantilever by chemical KOH etching and its surface analysis by TEM and AFM', Microsyst. Technol., 2015, Vol. 21, Iss. 3, pp. 661-668

[12] Mitwally, M.E, Tsuchiya, T., Tabata, O., Sedky, S.: 'Effect of localized $\mathrm{KrF}$ excimer laser treatment on fracture behaviors of freestanding $<110>$ and $<100>$ single crystal silicon beams', Microsyst. Technol., 2015, published online.

[13] Hajika, R., Yoshida, S., Kanamori, Y., Esashi, M., Tanaka, S.: 'An investigation of the mechanical strengthening effect of hydrogen anneal for silicon torsion bar', J. Micromech. Microeng., 2014, Vol. 24, Iss. 10, pp. 1-11.

[14] Uesugi, A., Hirai, Y., Sugano, K., Tsuchiya, T., and Tabata, O.: 'Effect of surface morphology and crystal orientations on tensile fracture property of (110) single crystal silicon', Transactions of JSME, Part A, 2013, Vol. 79, Iss. 804, pp. 1191-1200

[15] Weibull, W.: 'A statistical distribution function of wide applicability', J. Appl. Mech., 1951, Vol. 18, pp. 293-297

[16] Hesketh, P. J., Ju, C., Gowda, S., Zanoria, E., Danyluk, S.: 'Surface free energy model of silicon anisotropic etching', J. the Electrochemical Society, 1993, Vol. 140, Iss. 4, pp. 1080-1085

[17] Péreza, R., Gumbschb, P.: 'An AB initio study of the cleavage anisotropy in silicon', Acta mater., 2000, Vol. 48, pp. 4517-4530
[18] Aga, H., Nakano, M., and Mitani, K.: 'Study of HF defects in thin, bonded silicon-on-insulator dependent on original wafers, Jpn. J. Appl. Phys., Part 1, 1999, Vol. 38, Iss. 5, pp. 2694-2698 